\title{
Associação do envolvimento à actividade física e à aptidão em jovens madeirenses
}

\author{
Elisa C. Ramos ${ }^{1}$ \\ Duarte L. Freitas ${ }^{2}$ \\ José A. Maia ${ }^{3}$ \\ Gaston P. Beunen ${ }^{4}$ \\ Albrecht L. Claessens 4 \\ Élvio R. Gouveia 2 \\ António T. Marques 3 \\ Martine A. Thomis 4 \\ Johan A. Lefevre 4
}

https://doi.org/10.5628/rpcd.08.02.229

\author{
${ }^{1}$ Secretaria Regional de Educação e Cultura \\ Gabinete Coordenador do Desporto Escolar \\ Portugal \\ ${ }^{2}$ Departamento de Educação Física e Desporto \\ Universidade da Madeira \\ Portugal \\ ${ }^{3}$ Universidade do Porto, Faculdade de Desporto \\ Portugal \\ ${ }^{4}$ Department of Sport and Movement Sciences \\ Faculty of Kinesiology and Rehabilitation Sciences \\ Katholieke Universiteit Leuven, Bélgica
}

\section{RESUMO}

O objectivo central do presente estudo consistiu em investigar a associação do meio (urbano, semi-urbano e rural) à activida-de física e à aptidão na criança e no adolescente madeirense. A amostra foi constituída por 1498 sujeitos, 758 rapazes e 740 raparigas, que participaram no 'Estudo de Crescimento da Madeira'. A actividade física e a aptidão foram avaliadas através do questionário de Baecke e da bateria de testes motores Eurofit, respectivamente.

As crianças e adolescentes madeirenses do meio urbano apresentaram valores mais elevados de prática regular e sistemática de um ou mais desportos. Os resultados para as componentes da aptidão física não favorecem um único meio sócio-geográfico. Os rapazes do meio urbano e/ou semi-urbano foram mais proficientes na flexibilidade, força e resistência muscular, e potência, enquanto os rapazes rurais apresentaram melhores resultados na resistência aeróbia, força estática, e velocidade/agilidade. As raparigas do meio urbano e/ou semiurbano apresentaram melhores resultados na velocidade/agilidade, enquanto as raparigas do meio rural foram mais proficientes na força estática e na força e resistência muscular. A eliminação dos diferenciais negativos na actividade física e na aptidão associados ao meio sócio-geográfico irá resultar numa melhor saúde das crianças e adolescentes madeirenses.

Palavras-chave: actividade física, aptidão física, envolvimento, crianças, Portugal

\section{ABSTRACT \\ Environmental correlates of physical activity and fitness in youngsters from Madeira}

The main purpose of this study was to investigate the association between the area of residence (urban, semi-urban and rural) and physical activity and fitness in children and adolescents from Madeira island.

The sample included 1498 participants, 758 boys and 740 girls, of the 'Madeira Growth Study'. Physical activity was assessed by means of a questionnaire and physical fitness was measured with the Eurofit test battery.

Children and adolescents from urban areas showed higher values in regular sports and activities. Results for physical fitness do not favour one socio-geographic group. Boys from urban and semi-urban area's performed significantly better for flexibility, upper body muscular endurance, abdominal strength and power, whereas boys from the rural area performed better for aerobic endurance, static strength, running speed, agility and balance. Girls from urban and semi-urban area's obtained better results for running speed and agility, whereas girls from rural area performed better for static strength and upper body muscular endurance.

Programmes aimed at the elimination of urban-rural differences in physical activity and fitness, associated to socio-geographic area, should focus on increasing specific fitness levels, which might result in improved health conditions of children and adolescents living in Madeira.

Key-words: physical activity, physical fitness, environment, children, Portugal 


\section{INTRODUÇÃO}

Os níveis de actividade física e de aptidão na criança e no adolescente estão associados ou são influenciados pelas características de envolvimento(20).

As melhores condições de saúde e de alimentação no meio urbano são fundamentais para obter os níveis de energia exigidos para a actividade física regular. Contudo, a elevada densidade populacional, a falta de segurança e a inexistência de espaços de recreação e lazer podem contribuir para uma redução dos níveis de actividade física e aptidão no meio urbano.

Paralelamente, o meio rural está associado a um estilo de vida mais vigoroso e fisicamente activo(28, 23). A associação do meio (urbano, semi-urbano e rural) aos níveis de actividade física tem sido referida na literatura sem um padrão emergente. Os rapazes e raparigas Belgas $(26,25,36)$ da área rural apresentaram níveis de prática desportiva mais baixos do que os dos seus colegas da área urbana. Resultados similares foram encontrados em crianças e adolescentes Islandeses. Os sujeitos da área rural foram menos activos do que os seus pares da área urbana ${ }^{(17)}$. Em crianças Australianas, o contexto rural aumentou a probabilidade de participação nos clubes desportivos, enquanto as crianças urbanas integraram mais os 'clubes escola'(8). Ainda, Özdirenç et al.(22) observaram que a percentagem de crianças Turcas que não praticavam qualquer desporto foi mais elevada na área urbana comparativamente à área rural.

Os resultados da investigação acerca do diferencial nas componentes de aptidão física em função da área de residência são igualmente conflituosos.

Glanner(12) observou que os rapazes Brasileiros da área rural possuíam níveis de aptidão física mais elevados do que os dos seus colegas da área urbana. Em oposição, Renson et al.(26, 25) encontraram melhores desempenhos dos rapazes Belgas da área urbana em vários testes motores comparativamente aos colegas da área rural.

Em Portugal, Silva et al.(35) observaram que os adolescentes da área urbana do distrito de Coimbra dedicaram mais tempo às actividades físicas e desportivas, comparativamente aos seus pares dos grupos semi-urbanos e rurais. Similarmente, os rapazes da área rural apresentaram desempenhos mais fracos do que os dos seus colegas da área urbana e semiurbana na corrida de velocidade ( $25 \mathrm{~m})$, na impulsão vertical e no salto em comprimento sem corrida preparatória. Contrariamente aos resultados anteriores, Moreno e Vasconcelos(21) observaram que as crianças e adolescentes da área rural (Trás-os-Montes) foram mais proficientes do que os seus pares da área urbana (Matosinhos), mas as diferenças com significado estatístico foram limitadas ao 'shuttle run' (rapazes e raparigas) e à dinamometria manual (raparigas). Ainda, em rapazes Vianenses da área rural, Rodrigues et al.(27) observaram valores médios mais elevados nas provas de pull up, corrida de $50 \mathrm{~m}$, e 'shuttle run' de resistência, enquanto os seus pares da área urbana apresentaram melhores desempenhos no 'shuttle run' (velocidade/agilidade) e salto em comprimento sem corrida preparatória.

Os mecanismos através dos quais o meio exerce o seu impacto na actividade física e aptidão são poucos conhecidos $^{(13)}$. O desenvolvimento de intervenções para remover os gradientes demográficos associados à actividade física e aptidão pode depender de um entendimento claro de como é que factores modificáveis, tais como a prática desportiva, os estilos de vida sedentários e as influências sociais, actuam como mediadores do comportamento da criança e do adolescente ${ }^{(8)}$. Os dados do 'Estudo de Crescimento da Madeira' fornecem uma oportunidade única para quantificar esta associação. O objectivo da presente pesquisa foi investigar a associação entre o meio (urbano, semi-urbano e rural) e os níveis de actividade física e aptidão em crianças e adolescentes madeirenses.

\section{MATERIAL E MÉTODOS \\ Amostra}

As crianças e adolescentes que integram a amostra participaram no 'Estudo de Crescimento da Madeira'. No total, 1498 sujeitos (758 rapazes e 740 raparigas) entre os 7 e os 18 anos foram avaliados em 1996, 1997 e 1998 [o número de sujeitos por intervalo etário, sexo e meio (urbano, semi-urbano e rural) é apresentado no Quadro 1]. O 'Estudo de Crescimento da Madeira' apresenta um delineamento longitudinal misto com cinco coortes $(8,10,12$, 14 e 16 anos de idade) observados em intervalos anuais. Os elementos da amostra foram seleccionados de acordo com procedimentos estratificados proporcionais. A localização geográfica, o ano de escola- 
ridade e as características do edifício escolar foram os indicadores de estratificação. A amostra inclui 36 escolas da Região Autónoma da Madeira (RAM).

Quadro 1. Dimensão da amostra em função do intervalo etário, sexo e meio: urbano, semi-urbano e rural.

\begin{tabular}{lcccc}
\hline Idade (anos) & \multicolumn{3}{c}{ Meio } \\
\hline & Urbano & Semi-urbano & Rural & Total \\
\hline Rapazes & & & & \\
\hline $7-9$ & 67 & 70 & 42 & 179 \\
\hline $10-11$ & 68 & 52 & 28 & 148 \\
\hline $12-13$ & 74 & 61 & 26 & 161 \\
\hline $14-15$ & 93 & 36 & 24 & 153 \\
\hline $16-18$ & 91 & 12 & 14 & 117 \\
\hline Total & 393 & 231 & 134 & 758 \\
\hline Raparigas & & & & \\
\hline $7-9$ & 65 & 70 & 41 & 176 \\
\hline $10-11$ & 63 & 44 & 35 & 142 \\
\hline $12-14$ & 117 & 56 & 45 & 218 \\
\hline $15-18$ & 144 & 31 & 29 & 204 \\
\hline Total & 389 & 201 & 150 & 740 \\
\hline Grande Total & 782 & 432 & 284 & 1498 \\
\hline
\end{tabular}

\section{Delimitação do meio sócio-geográfico}

A delimitação do meio sócio-geográfico na RAM foi efectuada a partir da informação disponível no Instituto Nacional de Estatística (INE) ${ }^{(15)}$. Em traços gerais, o INE(15) ordenou os centros urbanos de Portugal Continental e da Região Autónoma da Madeira a partir do índice de centralidade, ie., a extensão das funções prestadas pelo lugar central, o grau de especialização e o número de unidade funcionais do centro urbano. A título de exemplo, em Portugal Continental, Lisboa apresenta o índice de centralidade mais elevado (34.34) e São Faustino, o índice de centralidade mais baixo (0.10). Os dados relativos aos 11 concelhos da RAM são apresentados no Quadro 2.

Os pontos de corte utilizados na delimitação do meio sócio-geográfico na RAM foram os seguintes: [1] meio urbano (índice de centralidade igual ou superior a 3.81), [2] semi-urbano (índice de centralidade compreendido entre 3.46 e 3.80), [3] rural (índice de centralidade compreendido entre $0 \mathrm{e}$ 3.45). Em termos de população, os intervalos são os seguintes: [1] meio urbano (número de residentes
Quadro 2. Índice de centralidade, 'rank', área e população residente nos 11 concelhos da RAM [adaptado do INE(15)].

\begin{tabular}{|c|c|c|c|c|}
\hline Meio/Concelho & IC & 'Rank' & Área $\left(\mathrm{km}^{2}\right]$ & PR \\
\hline \multicolumn{5}{|l|}{ Urbano } \\
\hline Funchal & 12.98 & 14 & 76 & 103961 \\
\hline \multicolumn{5}{|l|}{ Semi-urbano } \\
\hline Machico & 3.77 & 149 & 17 & 11947 \\
\hline Porto Santo & 3.55 & 172 & 40 & 4474 \\
\hline Câmara de Lobos & 3.52 & 176 & 23 & 30785 \\
\hline Santa Cruz & 3.46 & 184 & 14 & 6070 \\
\hline \multicolumn{5}{|l|}{ Rural } \\
\hline Ribeira Brava & 3.40 & 193 & 17 & 5941 \\
\hline São Vicente & 3.26 & 205 & 47 & 3336 \\
\hline Santana & 2.92 & 245 & 29 & 3439 \\
\hline Calheta & 2.89 & 247 & 21 & 3105 \\
\hline Ponta do Sol & 2.31 & 285 & 27 & 4224 \\
\hline Porto Moniz & 2.22 & 286 & 19 & 1700 \\
\hline
\end{tabular}

igual ou superior a 12001), [2] semi-urbano (intervalo compreendido entre 6000 e 12000 residentes) e [3] rural (intervalo compreendido entre 0 e 5999 residentes). De acordo com esta divisão, o meio urbano integra o concelho do Funchal; o meio semiurbano, os concelhos de Machico, Porto Santo, Câmara de Lobos e Santa Cruz; e o meio rural, os concelhos da Ribeira Brava, São Vicente, Santana, Calheta, Ponta do Sol e Porto Moniz.

\section{Actividade física}

O questionário desenvolvido por Baecke et al.(3) foi usado para avaliar a actividade física. O questionário de Baecke foi traduzido e culturalmente adaptado à população portuguesa $(39,10,9$, 34, 33). Embora a versão original apresente três dimensões (actividade física no trabalho, no desporto e nos tempos livres), o índice de trabalho não foi usado no presente estudo. Os itens de resposta são quantificados numa escala de 1 a 5 , excepto para as questões 1 e 9 . Foram calculados índices para o desporto (questões 9 a 12) e para os tempos livres (questões 13 a 16). O 'score' desportivo (prática regular e sistemática de um ou mais desportos) foi também calculado a partir da combinação da intensidade, tempo despendido por semana e proporção do ano com que essa modalidade foi realizada. 
Os membros da equipa de campo preencheram os questionários através de entrevista antes da avaliação antropométrica. As crianças com idades inferiores a 10 anos tiveram a ajuda do pai e/ou mãe ou do Professor de Educação Física. A fiabilidade teste-reteste para o índice desportivo e o índice dos tempos livres, avaliada com um intervalo de uma semana durante o estudo piloto, foram 0.80 e 0.73 , respectivamente. Estes resultados são similares aos encontrados por Baecke et al.(3) na amostra original (0.81 e 0.74 , respectivamente). Dados recentes sugerem que o questionário de Baecke e possíveis adaptações a este questionário fornecem informação válida e fiável da actividade física. Philippaerts et al.(24), verificaram que os coeficientes de correlação intra-classe para a actividade desportiva e a actividade nos tempos livres foram superiores a 0.70 em crianças e adolescentes de 12-18 anos. Em adição, a participação desportiva durante os tempos livres e índice desportivo mostraram uma correlação significativa $(\mathrm{R}>0.50)$ com os 'counts' do acelerómetro uniaxial 'Computer Science and Applications' (CSA).

\section{Aptidão física}

A aptidão física foi avaliada através da bateria de testes Eurofit( $(2)$, a qual inclui 9 testes relacionados com a saúde e a performance, nomeadamente: equilíbrio flamingo (equilíbrio), batimento em placas (velocidade dos membros superiores), 'sit and reach' (flexibilidade), salto em comprimento sem corrida preparatória (força explosiva), dinamometria manual (força estática), 'sit ups' (força do tronco), tempo de suspensão com os braços flectidos (força funcional) e 'shuttle run' 10x $5 \mathrm{~m}$ (velocidade e agilidade). $\mathrm{O}$ 'shuttle run' de resistência (corrida vaivém de $20 \mathrm{~m}$ ) foi substituído pela corrida/andar de 12 minutos (resistência cardio-respiratória) da bateria da AAHPERD(1). Esta opção é justificada pela forte herança cultural do teste de corrida/andar de 12 minutos na RAM. As considerações práticas incluem a popularidade do teste na determinação da aptidão aeróbia, a simplicidade de administração do teste e o número elevado de sujeitos que podem ser avaliados num curto espaço de tempo.

A fiabilidade dos testes motores foi igualmente verificada no estudo piloto. Os coeficientes de correlação intra-classe (R) estavam compreendidos entre 0.78 (salto em comprimento sem corrida preparatória) e 0.96 (dinamometria manual).

\section{Procedimentos estatísticos}

Todas as análises foram efectuadas a partir do recurso ao 'Statistical Analysis System Program'(32). As esta- tísticas descritivas usadas foram a média e o desvio padrão $(M \pm D P)$. A fiabilidade teste-reteste para o índice desportivo e o índice dos tempos livres foi estimada na base do coeficiente de correlação momento-produto de Pearson (r). Para os testes motores utilizamos o coeficiente de correlação intraclasse (R). O significado estatístico da diferença de médias nos indicadores da actividade física e da aptidão em função do meio (urbano, semi-urbano e rural) foi determinado a partir da análise de variância (ANOVA). O teste de Tukey foi utilizado nas comparações múltiplas 'post hoc' sempre que o valor de $\mathrm{F}$ evidenciou significado estatístico. Os resultados foram considerados estatisticamente significativos quando $\mathrm{p}<0.05$.

\section{RESULTADOS}

A representação gráfica dos resultados para a actividade física ('score' desportivo, índice desportivo e índice dos tempos livres) em função do meio sóciogeográfico é apresentada na Figura 1a-c. Globalmente, os rapazes e raparigas do meio urbano apresentam valores médios de prática desportiva regular e sistemática ('score' desportivo) mais elevados do que os seus pares do meio semi-urbano e/ou rural. Diferenças com significado estatístico são observadas aos 12-13 anos, nos rapazes, e aos 7-9 anos, nas raparigas (Figura 1a). Para o índice desportivo são visíveis duas diferenças com significado estatístico. Os rapazes do meio urbano apresentam valores mais elevados do que os dos colegas do meio rural (7-9 anos) e semi-urbano (12-13 anos). O diferencial nas raparigas é muito reduzido (Figura $1 b$ ). As diferenças associadas ao meio no índice dos tempos livres são igualmente pequenas. Contudo, os rapazes e as raparigas do meio rural tendem a apresentar valores médios mais elevados do que os do meio urbano e semi-urbano. Nos rapazes, as diferenças com significado estatístico são observadas aos 14-15 e 16-18 anos, enquanto nas raparigas as diferenças são visíveis aos 7-9 anos (Figura 1c).

As Figuras 1d, 2 e 3 procedem à ilustração gráfica dos valores médios nos testes motores em função do meio sócio-geográfico (urbano, semi-urbano e rural). Os rapazes do meio rural apresentam valores médios na corrida/andar de 12 minutos (resistência cardiorespiratória) ligeiramente superiores aos seus colegas do meio urbano e/ou semi-urbano. As diferenças assumem significado estatístico aos 7-9 anos. Nas raparigas, os valores são muito similares entre os meios (Figura 1d). O diferencial no 'sit and reach' 
Figura 1. 'Score' desportivo (a), índice desportivo (b), índice dos tempos livres (c) e corrida/andar de 12 minutos dos rapazes e raparigas madeirenses classificados em três meios sócio-geográficos: urbano ( $\mathbf{0}$ ), semi-urbano ( $\boldsymbol{\Delta}$ ) e rural ( $\bullet$ ].

a

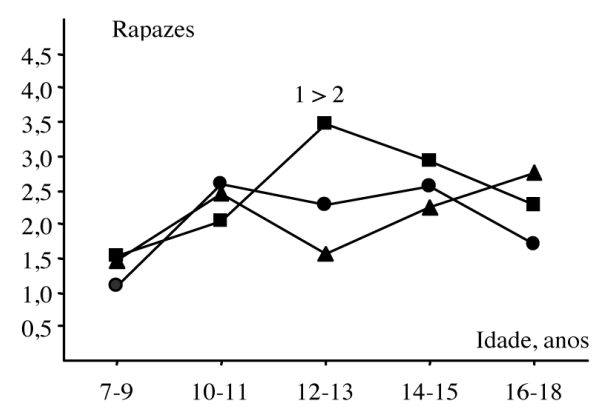

'Score' desportivo

b

Índice desportivo
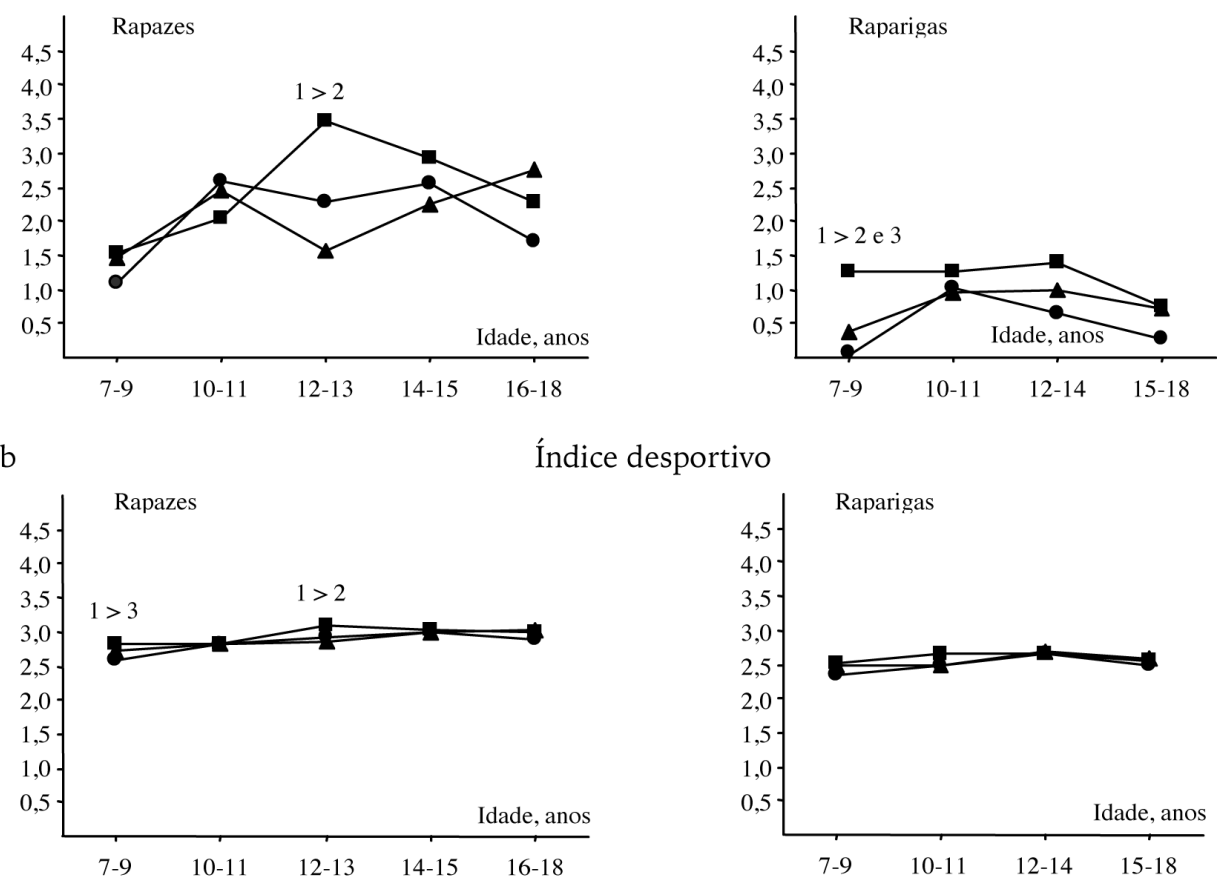

Índice dos tempos livres

c
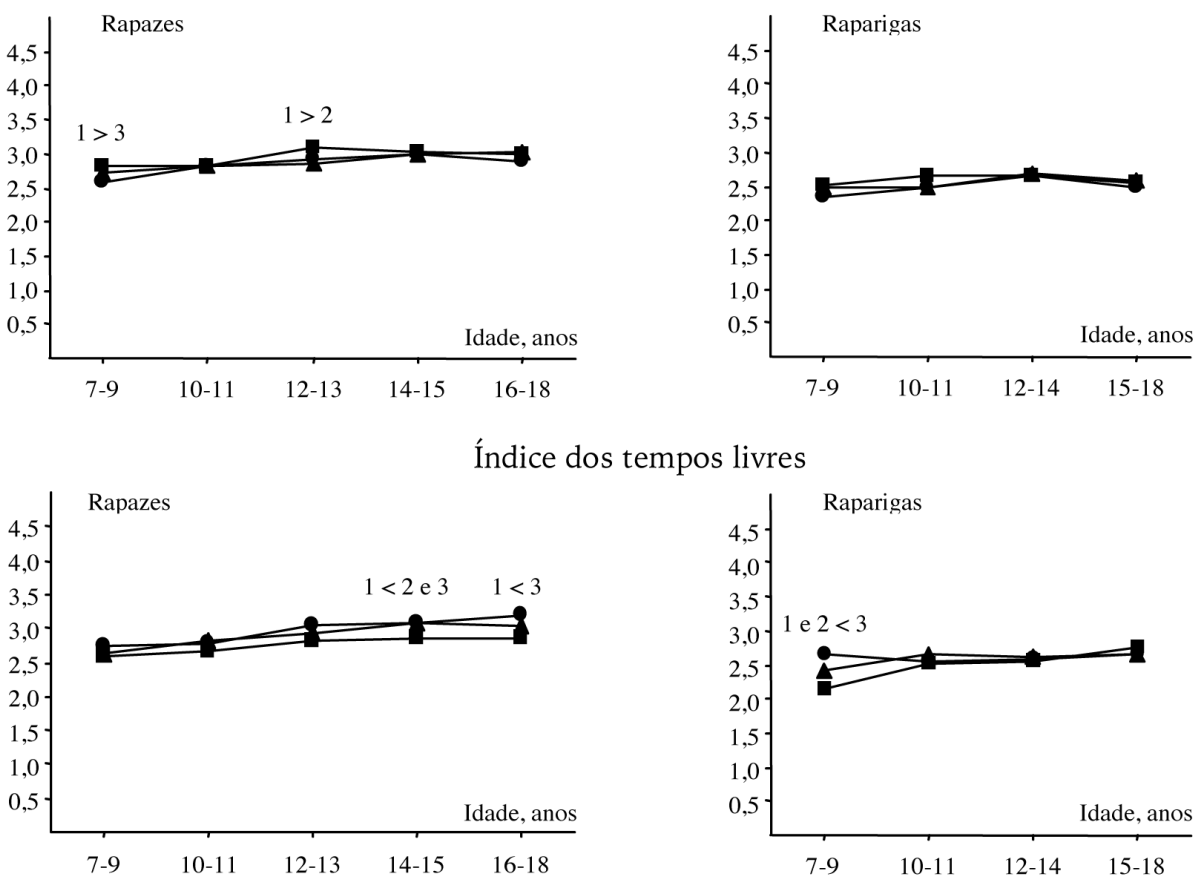

d

Corrida/andar de 12 minutos
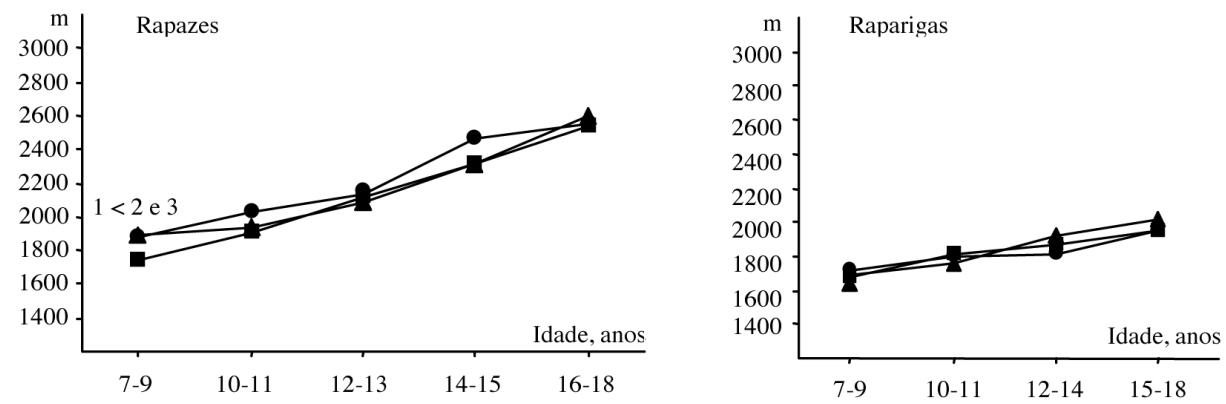
Figura 2. Testes motores: 'Sit and reach' (a), tempo de suspensão com os braços flectidos (b), 'sit ups' e dinamometria manual (d) dos rapazes e raparigas madeirenses classificados em três meios sócio-geográficos: urbano ( $\mathbf{\square}$ ), semi-urbano ( $\boldsymbol{\Delta}$ ) e rural ( $\bullet$ ).
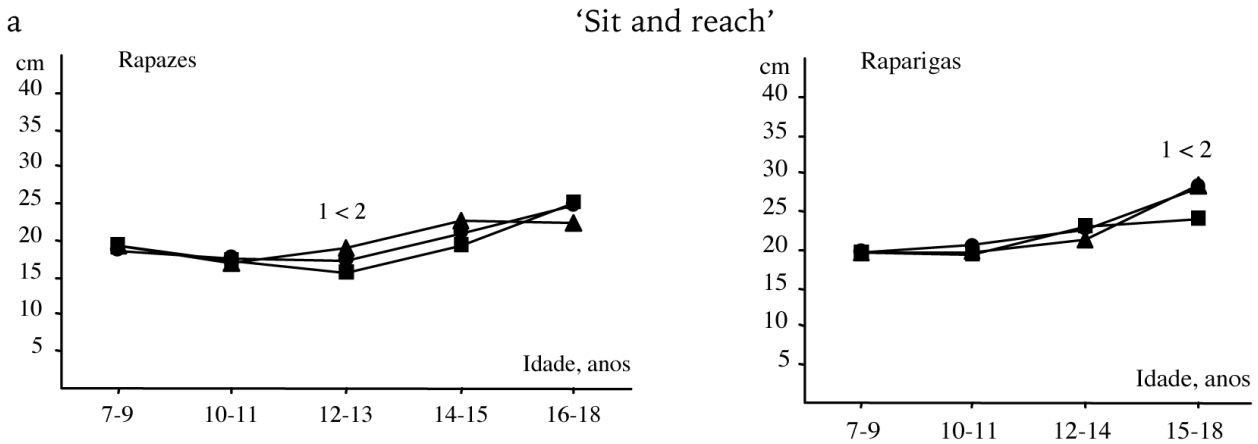

$\mathrm{b}$

Tempo de suspensão com os braços flectidos
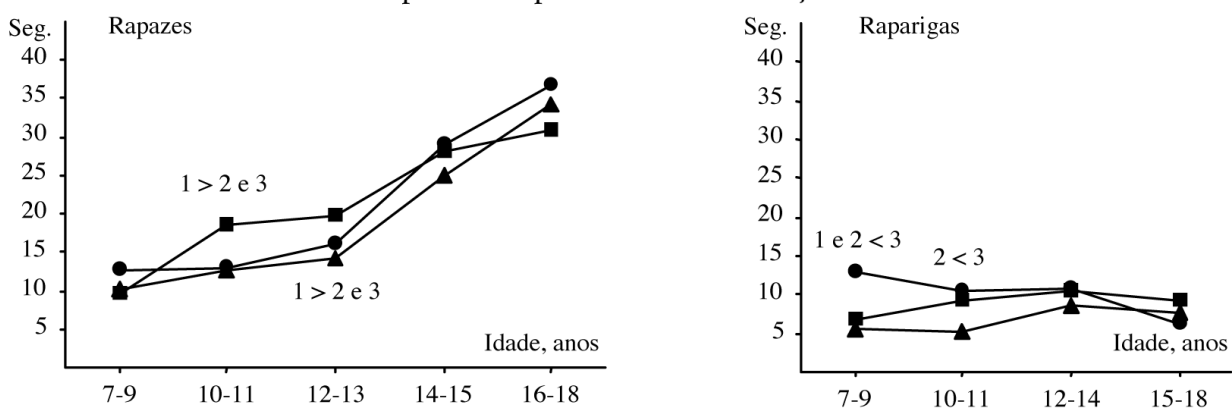

c

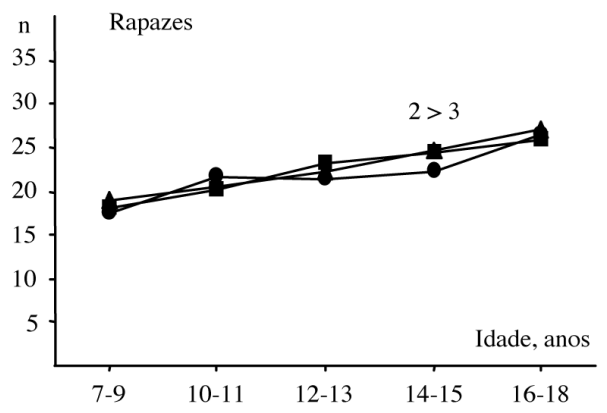

'Sit ups'

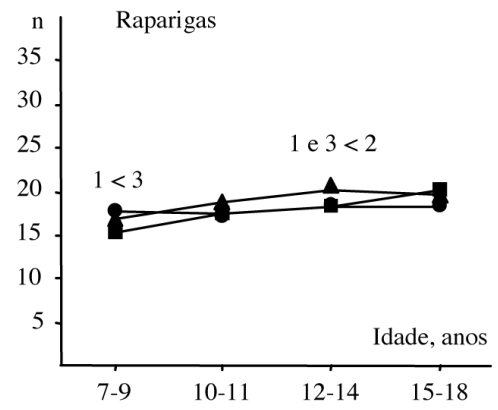

d

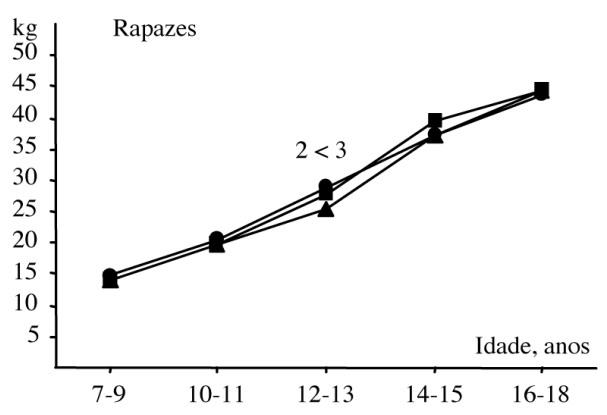

Raparigas

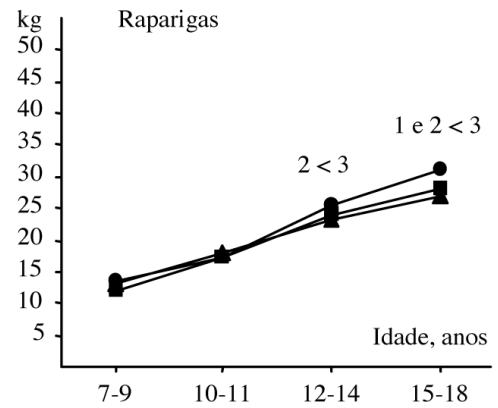


Figura 3. Testes motores: Salto em comprimento sem corrida preparatória (a), 'shuttle run' (b), batimento em placas (c) e equilíbrio flamingo (d) dos rapazes e raparigas madeirenses classificados em três meios sócio-geográficos: urbano ( $\square$ ), semi-urbano ( $\mathbf{\Delta}$ ) e rural ( $\bullet$ ).

a

Salto em comprimentos sem corrida preparatória
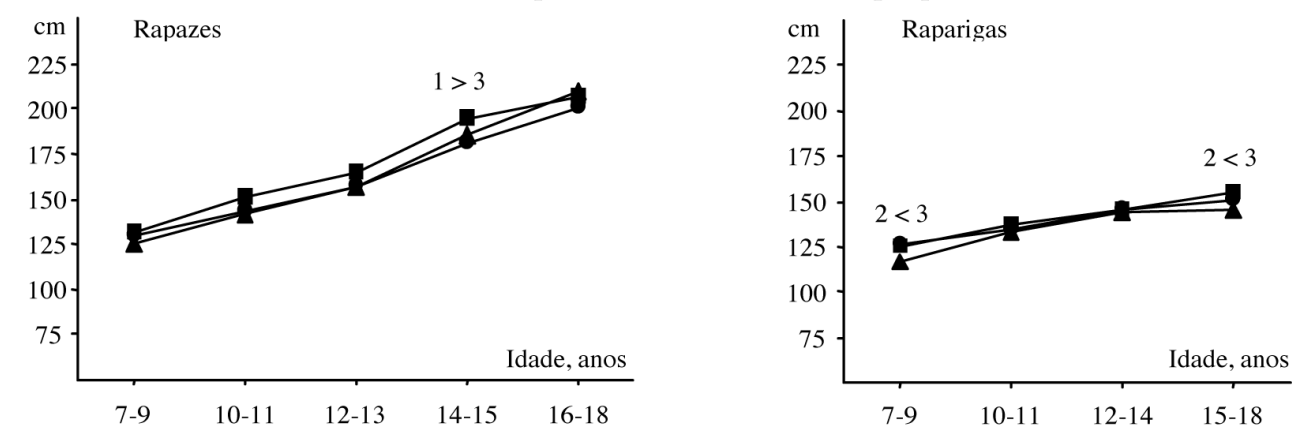

b

'Shuttle run'
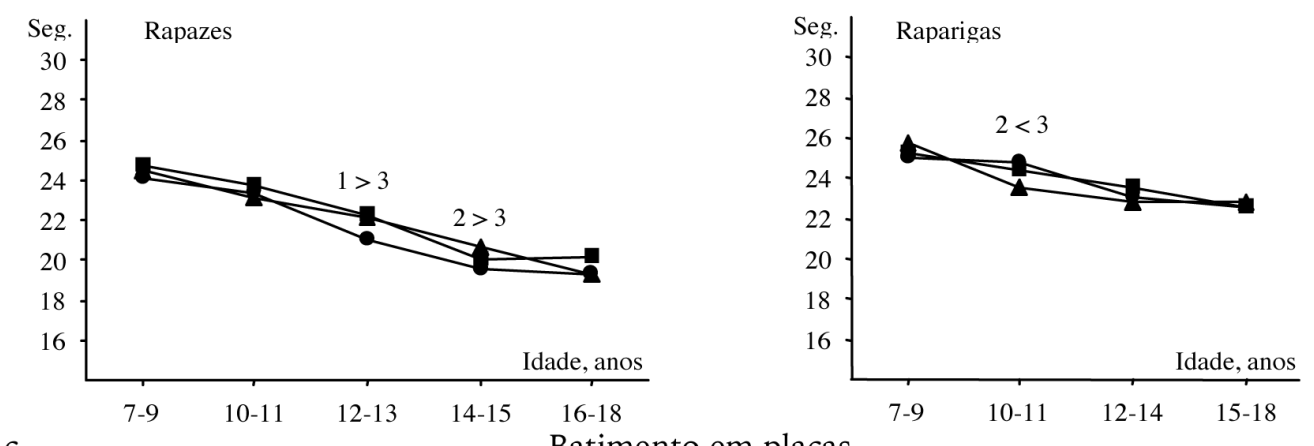

C

Batimento em placas
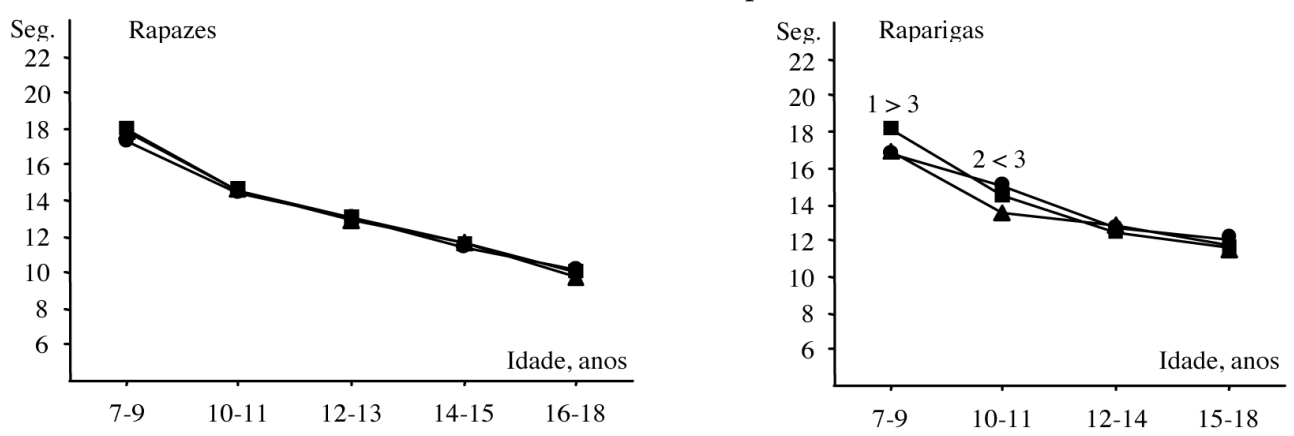

d

Equilíbrio Flamingo
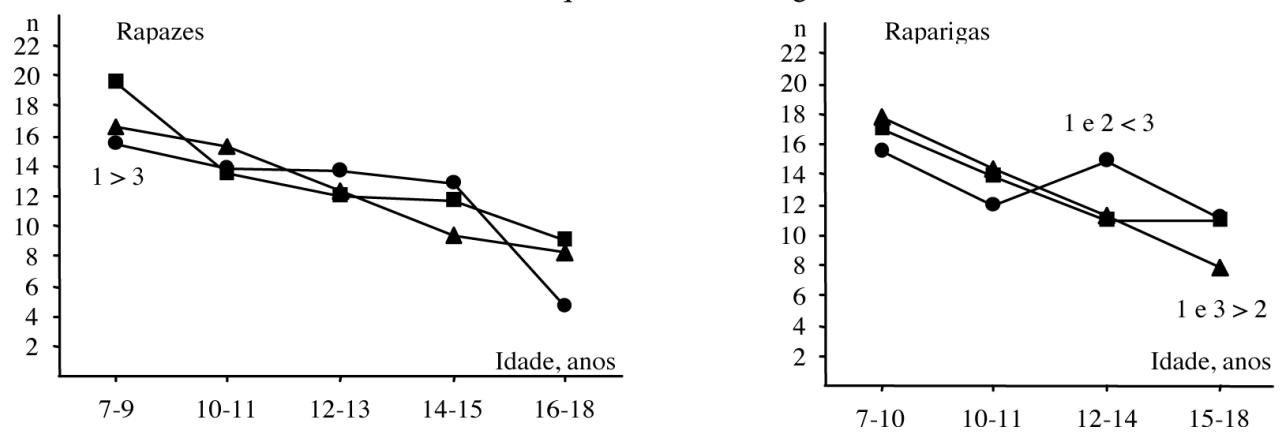
(flexibilidade) é favorável ao meio semi-urbano, nos rapazes (12-13 anos), e nas raparigas (15-18 anos), comparativamente ao meio urbano (Figura 2a). Para o tempo de suspensão com os braços flectidos (força funcional), os rapazes do meio urbano apresentam melhores desempenhos do que os do meio semi-urbano e/ou rural aos 10-11 e 12-13 anos. As diferenças para as raparigas são significativas aos 7-9 e 10-11 anos favorecendo o meio rural (Figura 2b). As diferenças associadas ao meio no 'sit ups' (força abdominal) são inconsistentes. Os rapazes do meio semi-urbano (14-15 anos) são mais proficientes do que os colegas do meio rural, enquanto as raparigas do meio rural (7-9 anos) e do meio semi-urbano (12-14 anos) são mais fortes do que os seus pares do meio urbano (Figura 2c). Ao nível da dinamometria manual (força estática), diferenças com significado estatístico são observadas nos rapazes (12-13 anos) e nas raparigas (12-14 e 15-18 anos). Em ambos, rapazes e raparigas, o meio rural é mais forte do que o meio urbano e/ou semi-urbano (Figura 2d). O desempenho no salto em comprimento sem corrida preparatória (força explosiva dos membros inferiores) também difere com o meio. O diferencial é mais evidente nos rapazes: o meio urbano é mais proficiente do que o meio semi-urbano e rural no intervalo 7-15 anos. No entanto, diferenças com significado estatístico são apenas observadas aos 14-15 anos. Nas raparigas, observamos um padrão distinto: o meio rural apresenta melhores desempenhos do que o meio semi-urbano aos 7-9 anos, enquanto o meio urbano apresenta melhores resultados do que o meio semi-urbano aos 15-18 anos (Figura 3a). As diferenças associadas ao meio no 'shuttle run' (velocidade/agilidade) são geralmente favoráveis ao meio rural, nos rapazes. Diferenças com significado estatístico são observadas aos 12-13 e 14-15 anos. Nas raparigas, o meio semi-urbano é mais proficiente aos 10-11 e 12-14 anos, mas o diferencial alcança significado estatístico apenas aos 10-11 anos (Figura 3b). Para o batimento em placas (velocidade de movimento dos membros superiores) os valores médios dos rapazes urbanos, semi-urbanos e rurais são quase coincidentes. Em oposição, as raparigas do meio rural são mais proficientes do que as suas colegas do meio urbano aos 7-9 anos. Aos 10-11 anos, as raparigas do meio semi-urbano apresentam melhores desempenhos do que os pares do meio rural (Figura 3c). Os rapazes do meio rural (7-9 anos) são mais proficientes do que os seus colegas do meio urbano no teste de equilíbrio flamingo (equilíbrio). Pelo contrá- rio, as raparigas do meio urbano e semi-urbano (1214 anos) apresentam melhores desempenhos do que as colegas do meio rural. Aos 15-18 anos, as raparigas do meio semi-urbano são mais proficientes do que os pares do meio urbano e rural (Figura 3d).

\section{DISCUSSÃO}

O meio (urbano, semi-urbano e rural) está associado à prática regular e sistemática de um ou mais desportos ('score' desportivo) favorecendo as crianças e adolescentes madeirenses do meio urbano. A diferença no índice desportivo é reduzida, mas os rapazes do meio urbano apresentam valores médios mais elevados nos intervalos etários iniciais. No índice dos tempos livres, os rapazes do meio semi-urbano e rural apresentam valores médios de actividade física mais elevados. Os resultados para as componentes da aptidão física não favorecem um único meio sócio-geográfico.

Os nossos resultados são consistentes com um trabalho anterior realizado em Portugal continental. Os adolescentes Conimbricenses do meio urbano dedicaram mais tempo às actividades físicas e desportivas, comparativamente aos seus pares do meio semiurbano e rural(35). Descobertas similares, mostrando que os rapazes e/ou raparigas do meio urbano apresentaram níveis mais elevados de prática desportiva do que aqueles dos meios semi-urbano e/ou rural, foram encontradas na Bélgica $(26,25,36)$ e na Islândia(17). Contrariamente a esta linha de resultados, Dolmman et al.(8) observaram que o contexto rural aumentou a probabilidade de participação das crianças Australianas nos clubes desportivos em aproximadamente $61 \%$ nos rapazes e $44 \%$ nas raparigas. Similarmente, a percentagem de crianças Turcas que não estava envolvida em qualquer actividade desportiva foi de $35 \%$, no meio urbano, e de $30.6 \%$ no meio rural(22). Há, pois, alguma inconsistência na literatura acerca desta associação.

Uma possível explicação para estes resultados poderá residir num padrão de exercício melhor definido pelas crianças e adolescentes do meio urbano(31), numa maior variedade de facilidades para o exercício $(28,30)$ e numa maior ênfase no desporto infantojuvenil(17). Isto poderá ocorrer na RAM, embora não se possua informação que documente os estilos de vida da criança e do adolescente madeirense. Uma outra descoberta interessante é o declínio no 'score' desportivo depois dos 12-13 anos nos rapazes, à excepção do meio semi-urbano, e a partir dos 12-14 anos nas raparigas. Este decréscimo poderá reflectir a 
mudança de interesses e expectativas, a pressão dos colegas e a falta de motivação, à medida que os jovens percorrem a puberdade(20). Paralelamente, os rapazes madeirenses do meio urbano apresentaram valores médios no índice desportivo mais elevados do que os seus pares do meio semi-urbano e/ou rural, em alguns intervalos etários. As justificações anteriores poderão explicar este diferencial. Contudo, no índice dos tempos livres, são os rapazes e raparigas do meio rural que apresentaram valores médios mais elevados, embora as diferenças não assumissem significado estatístico, na maioria dos intervalos etários. Há, também, justificações para estes resultados.

Primeiro, o meio urbano apresenta uma elevada densidade populacional e os espaços de recreação e lazer são reduzidos $(28,23)$. Segundo, as condições de segurança são fracas no meio urbano(22). Terceiro, as crianças e adolescentes do meio urbano despendem mais tempo a ver televisão ou a jogar no computador(40). De igual modo, Silva et al.(35) observaram que os jovens Portugueses do meio urbano frequentavam mais cafés e centros comerciais.

A segunda relação refere-se à variação nos níveis de aptidão física associada ao meio urbano, semi-urbano e rural. Os rapazes madeirenses do meio rural apresentaram valores médios na corrida/andar de 12 minutos ligeiramente superiores aos seus pares do meio urbano e/ou semi-urbano. Os nossos resultados são paralelos à proficiência na corrida de 8 minutos das crianças Mexicanas, aos 7-9 anos(23). Um diferencial favorável ao meio rural foi igualmente observado em crianças e/ou adolescentes Australianos $^{(8)}$, Belgas(36), Brasileiros(11, 12) e Polacos(40). Contudo, Moreno e Vasconcelos(21) não observaram diferenças com significado estatístico entre os meios urbano e rural na corrida de 12 minutos em adolescentes Portugueses. A ausência de diferenças com significado estatístico entre os meios urbano e rural foi também observada em crianças e/ou adolescentes Alemães $(28,18)$ e Turcos(22).

A diferença urbano-rural na resistência cardio-respiratória foi atribuída à actividade física em adolescentes Japoneses. Tamura(37) observou que os sujeitos do meio rural andaram a pé distâncias relativamente mais longas para se dirigirem para a escola e que estes despenderam mais tempo na actividade física. Os resultados da presente pesquisa não confirmam esta tendência. Paralelamente, é conhecido que o peso e a gordura corporal têm uma influência negativa nos itens da aptidão física que requerem o deslo- camento do corpo(20). Dados do 'Estudo de Crescimento da Madeira' revelaram que os rapazes do meio rural são mais leves do que os seus pares do grupo urbano aos 14-15 anos. Similarmente, o diferencial nas pregas de adiposidade subcutânea associado ao meio sócio-geográfico foi reduzido nos rapazes (dados não apresentados). Assim, o peso corporal e as pregas de adiposidade subcutânea não explicam os resultados ligeiramente superiores dos rapazes do meio rural. Contudo, este diferencial poderá, entre outros factores, ser interpretado pela maior familiarização com o teste, maior economia de esforço e uma atitude positiva relativamente à testa$\operatorname{gem}^{(29,8)}$.

A associação do meio ao teste 'sit and reach' (flexibilidade) não foi muito expressiva na amostra madeirense. Resultados similares foram encontrados no distrito de Viana do Castelo, em rapazes dos 7 aos $10 \operatorname{anos}^{(27)}$ e em adolescentes, 15-18 anos, do distrito de Coimbra ${ }^{(35)}$. No entanto, valores médios mais elevados no meio rural, comparativamente ao meio urbano, foram encontrados no $\operatorname{Brasil}(11,12)$, na Polónia(40), em Portugal(21) e na Turquia(22). Um diferencial favorável ao meio urbano foi também observado na Bélgica(36) e no México(23).

Contrariamente a alguns estudos anteriores $(36,18,11$, 21, 22), o meio urbano (rapazes) e o meio semi-urbano (raparigas) apresentaram valores mais elevados no tempo de suspensão com os braços flectidos (força funcional) na amostra madeirense. Ao nível do 'sit ups' (força e resistência abdominal), o meio semi-urbano apresentou uma tendência para valores mais elevados, comparativamente ao meio urbano e/ou rural. Os nossos resultados são consistentes com os dos seus congéneres Mexicanos(23) e Polacos ${ }^{(40)}$. Em oposição, a ausência de diferenças ou valores médios favoráveis ao meio rural foram observados na Alemanha(18), no Brasil(11, 12), na Polónia(40), em Portugal(35, 27) e na Turquia(22). No salto em comprimento sem corrida preparatória (força explosiva dos membros inferiores), os nossos resultados são convergentes com muitas das pesquisas acima referidas, particularmente nas crianças e adolescentes do sexo masculino.

A actividade física pode ajudar a interpretar os nossos resultados. No contexto do tempo de suspensão com os braços flectidos, o melhor desempenho dos rapazes do meio urbano (12-13 anos) poderá ser explicado por valores mais elevados no 'score' desportivo e no índice desportivo. As raparigas do meio rural (7-9 anos) apresentaram, também, um índice 
dos tempos livres superior aos seus pares do meio urbano e semi-urbano. De igual modo, o peso corporal poderá esclarecer parte da variação encontrada na amostra madeirense. Quanto mais elevado é o peso corporal menor é o desempenho nos testes motores que requerem a projecção e elevação do corpo(20). Esta análise vai ao encontro dos resultados das raparigas madeirenses do meio semi-urbano, no intervalo etário 7-9 anos. O peso foi mais elevado e, consequentemente, menor o desempenho no tempo de suspensão com os braços flectidos. Uma interpretação similar poderá realizar-se para as raparigas do meio semi-urbano no salto em comprimento sem corrida preparatória.

Ainda no salto em comprimento sem corrida preparatória, a tendência para valores médios mais elevados dos rapazes do meio urbano poderá ser entendida como uma melhor técnica de execução.

Observação idêntica foi efectuada por Peña Reyes et al.(23) em crianças e adolescentes Mexicanos. Uma completa e eficiente acção dos membros superiores e dos membros inferiores, com uma ligeira inclinação do tronco à frente no momento da impulsão, parece resultar em distâncias mais longas de salto(7). Analogamente, no teste 'sit ups', os melhores desempenhos do meio urbano e/ou semi-urbano podem ser interpretados pela maior familiarização com o teste. Situação similar foi observada em crianças e adolescentes Brasileiros(12).

Ao nível da dinamometria manual (força estática), os valores médios mais elevados do meio rural, nos rapazes (12-13 anos) e nas raparigas (12-14 e 15-18 anos), são paralelos aos resultados encontrados em crianças de Trás-os-Montes(21). Pelo contrário, resultados favorecendo o meio urbano e/ou semi-urbano foram observados na Polónia(40). Na literatura, os valores mais elevados na dinamometria manual dos rapazes e raparigas do meio rural são justificados pelas tarefas laborais desenvolvidas, nomeadamente, alimentar os animais, apanhar erva e utilizar instrumentos na lavoura $(21,23)$.

O tamanho corporal e o estatuto maturacional podem, também, ser justificativos de alguns dos resultados observados no domínio da força. Os rapazes de maturação avançada são mais fortes do que os seus pares da mesma idade cronológica, mas de maturação atrasada $(6,4)$. As raparigas de maturação avançada são igualmente mais fortes do que as suas colegas de maturação atrasada no início da adolescência (11-15 anos), mas o diferencial tende a diminuir aos 16-17 anos(16, 5). Dados do 'Estudo de
Crescimento da Madeira' revelaram que os rapazes do meio urbano (14-15 anos) estavam avançados na maturação esquelética [método Tanner-Whitehouse II(38) ] e foram mais altos, comparativamente aos seus colegas do meio semi-urbano e rural. Nas raparigas, não foi observado um diferencial na maturação esquelética em função do meio (dados não apresentados). Deste modo, o maior tamanho corporal e o avanço maturacional dos rapazes, aos 14-15 anos, poderá explicar os melhores desempenhos no 'sit ups' e no salto em comprimento sem corrida preparatória.

No teste 'shuttle run', os melhores desempenhos dos rapazes madeirenses do meio rural foram também observados em adolescentes de Trás-os-Montes (21). A maior proficiência das raparigas do meio semiurbano é consistente com os resultados encontrados em adolescentes Polacas (40). O tamanho corporal poderá justificar o diferencial observado na RAM. Malina ${ }^{(19)}$ refere que os grupos de menor estatura apresentam performances mais fracas nas tarefas que requerem velocidade e potência devido à sua menor massa muscular. Aos 10-11 anos, as raparigas do meio semi-urbano são mais altas do que as suas colegas do meio rural (dados não apresentados). Isto é, o melhor desempenho do grupo semi-urbano poderá ter por base a sua maior altura e, provavelmente, mais massa isenta de gordura. De igual modo, os melhores desempenhos dos rapazes do meio rural, aos 14-15 anos, podem ser interpretados pelos valores mais elevados no índice dos tempos livres. Moreno e Vasconcelos(21) explicaram os melhores desempenhos das crianças e adolescentes do meio rural pela maior quantidade de jogos e movimento.

A ausência de diferenças com significado estatístico no desempenho do teste de batimento em placas, pelos rapazes do meio urbano, semi-urbano e rural é corroborada em pesquisas desenvolvidas na

Turquia(22) e em Portugal continental(21). No sexo feminino, o melhor desempenho do meio semi-urbano, aos 10-11 anos, foi também observado na Polónia(40). Na mesma linha de análise, o número reduzido de diferenças com significado estatístico no teste de equilíbrio flamingo encontrado nos rapazes madeirenses, é paralelo aos seus pares de Portugal continental(21). Nas raparigas, os melhores desempenhos do meio urbano e semi-urbano são consistentes com os resultados encontrados na Polónia(40). Em suma, as crianças e adolescentes madeirenses do meio urbano apresentam uma tendência para valores 
médios mais elevados de prática regular e sistemática de uma ou mais modalidades desportivas. Em oposição, os rapazes do meio semi-urbano e rural apresentam valores médios de actividade física ligeiramente mais elevados nos tempos livres. Apenas $33 \%$ (rapazes) e $16.6 \%$ (raparigas) das diferenças de médias nos indicadores da actividade física em função do meio (urbano, semi-urbano e rural) alcançam significado estatístico. A associação entre o meio e as componentes da aptidão física é inconsistente. Resultados parcelares, ie., considerando apenas alguns intervalos etários, fornecem indicações que os rapazes do meio urbano e/ou semi-urbano são mais proficientes no 'sit and reach', no tempo de suspensão com os braços flectidos, no 'sit ups' e no salto em comprimento sem corrida preparatória. Os rapazes do meio rural apresentam melhores desempenhos na corrida/andar de 12 minutos, na dinamometria manual, no 'shuttle run' e no equilíbrio flamingo. As raparigas do meio urbano e/ou semi-urbano apresentam melhores resultados no 'shuttle run', enquanto as raparigas do meio rural são mais proficientes na dinamometria manual e no tempo de suspensão com os braços flectidos. Ainda nas raparigas, é observado um padrão irregular no 'sit and reach', no 'sit ups', no salto em comprimento sem corrida preparatória, no batimento em placas e no equilíbrio flamingo. Globalmente, $22.2 \%$ (rapazes) e $38.9 \%$ (raparigas) das diferenças nos testes de aptidão física em função do meio (urbano, semi-urbano e rural) alcançam significado estatístico.

\section{LOCAL DE REALIZAÇÃO DO ESTUDO}

$O$ presente estudo foi realizado na Universidade da Madeira, Departamento de Educação Física e Desporto, Campus Universitário da Penteada, 9000390 Funchal

\section{CORREPONDÊNCIA}

\section{Duarte Luís de Freitas}

Universidade da Madeira

Departamento de Educação Física e Desporto

Campus Universitário da Penteada

9000-390 Funchal

Portugal-Madeira

Tel. 291705332 Fax. 291705339

E-mail:dfreitas@uma.pt

\section{REFERÊNCIAS}

1. AAHPERD (1988). The AAHPERD Physical Best Program. Reston, VA: American Alliance for Health, Physical Education, Recreation and Dance.

2. Adam C, Klissouras V, Ravassolo M, Renson R, Tuxworth W, Kemper H, Van Mechelen W, Hlobil H, Beunen G, Levarlet-Joye H, Van Lierde A (1988). Eurofit. Handbook for the Eurofit Test of Physical Fitness. Rome: Council of Europe. Committee for the Development of Sport.

3. Baecke J, Burema J, Frijters J (1982). A short questionnaire for the measurement of habitual physical activity in epidemiological studies. Am J Clin Nutr 36: 936-942.

4. Beunen G, Malina R, Van't Hof M, Simons J, Ostyn M, Renson R, Van Gerven D (1988). Adolescent growth and motor performance. A longitudinal study of Belgian boys. HKP Sport Science Monograph Series. Champaign, IL: Human Kinetics.

5. Beunen G, Ostyn M, Renson R, Simons J, Van Gerven D (1976). Skeletal maturation and physical fitness of girls aged 12 through 16 . Hermes X: 445-457.

6. Beunen G., Ostyn M, Simons J, Renson R, Van Gerven D (1981). Chronological and biological age as related to physical fitness in boys 12 to 19 years. Ann Hum Biol 8(4): 321-331.

7. Clark J, Phillips S (1985). A developmental sequence of the standing broad jump. In: Clark J, Humphrey J (eds). Motor Development, Current Selected Research. Princeton: Princeton Book Co.

8. Dollman J, Norton K, Tucker G (2002). Anthropometry, fitness and physical activity of urban and rural south Australian children. Pediatric Exercise and Science 14: $297-$ 312.

9. Freitas D, Maia J, Beunen G, Claessens A, Thomis M, Marques A, Crespo M, Lefevre J (2007). Socio-economic status, growth, physical activity and fitness: The Madeira Growth Study. Ann Hum Biol 34: 107-122.

10. Freitas DL, Maia JA, Beunen GP, Lefevre JA, Claessens AL, Marques AT, Rodrigues AL, Silva CA, Crespo MT (2002) Crescimento somático, maturação biológica, aptidão física, actividade física e estatuto sócio-económico de crianças e adolescentes madeirenses. O Estudo de Crescimento da Madeira. Funchal: Universidade da Madeira.

11. Glanner MF (2002). Nível de atividade física e aptidão física relacionada à saúde em rapazes rurais e urbanos. Ver Paul Educ Fís 16(1): 76-85.

12. Glanner MF (2005). Aptidão física relacionada à saúde de adolescentes rurais e urbanos em relação a critérios de referência. Ver Bras Educ Fís 19(1): 13-24.

13. Gordon-Larsen, P, McMurray R, Popkin B (2000). Determinants of adolescent physical activity and inactivity patterns. Pediatrics 105(6): 83-95.

14. Gouveia ER, Freitas DL, Maia JA, Beunen GP, Claessens AL, Marques AT, Thomis MA, Almeida SM, Levefre JA (2007). Prevalência de excesso de peso e de obesidade em crianças e adolescentes da Região Autónoma da Madeira, Portugal. Acta Pediátrica Portuguesa (submetido).

15. Instituto Nacional de Estatística (2004). Sistema urbano: áreas de influência e marginalidade funcional. Região Autónoma da Madeira / Instituto Nacional de Estatística. Lisboa: INE.

16. Jones H (1949). Motor performance and growth: a developmental study of static dynamometric strength. Berkeley: University 
of California Press.

17. Kristjansdottir G, Vilhjolmsson R (2001).

Sociodemographic differences in patterns of sedentary and physically active behaviour in older children and adolescents. Acta Paediatr 90: 29-35.

18. Krombholz H (1997). Physical performance in relation to age, sex, social class and sports activities in kindergarten and elementary school. Perceptual and Motor Skills 84: 11681170.

19. Malina R (1986). Motor development and performance of children and youth in undernourished populations. In: Katch F (ed.). Sport, Health and Nutrition. Champaign, IL: Human Kinetics, 213-225.

20. Malina R, Bouchard C, Bar-Or O (2004). Growth, maturation, and physical activity. Champaign, IL: Human Kinetics.

21. Moreno D, Vasconcelos O (2003). Motor performance and maturational status. Study in children of two different school environments (rural and urban). Revista Portuguesa de Ciências do Desporto S3(2): 163-165.

22. Özdirenç M, Ozcan A, Akin F, Gelecek N (2005). Physical fitness in rural children compared with urban children in Turkey. Pediatrics International 47(1): 26-31.

23. Peña-Reyes M, Tan S, Malina R (2003). Urban-rural in the physical fitness of school children in Oaxaca, Mexico. Ann Hum Biol 15: 800- 813.

24. Philippaerts R, Matton L, Wijndaele K, Balduck A, De Bourdeaudhuij I, Lefevre J (2005). Validity of a physical activity computer questionnaire in 12- to 18-year-old boys and girls. Int J Sports Med 26: 1-6.

25. Renson R, Beunen G, De Witte L, Ostyn M, Simons J, Van Gerven e D (1980). The social spectrum of the physical fitness of 12 to 19 year-old boys. In: Ostyn M, Beunen G, Simons J (eds). Kinanthropometry II. International Series on Sport Sciences. Baltimore: University Park Press. 9: 105-118.

26. Renson R, Beunen G, Ostyn M, Simons J, Swalus P, Van Gerven D (1978). Social differentiation of physical fitness of preadolescent Belgian boys. In: Shephard R, Lavallée $\mathrm{H}$ (eds). Physical Fitness Assessment. Principles, Practice and Application. Illinois: Charles C. Thomas Publisher, 248-256.

27. Rodrigues L, Bezerra P, Saraiva L (2005). Influência do meio (urbano e rural) no padrão de aptidão física de rapazes de Viana do Castelo, Portugal. Revista Portuguesa de Ciências do Desporto 5(1): 77-84.

28. Rutenfranz J, Lange Andersen K, Seliger V, Masironi R (1982). Health standards in terms of exercise fitness of school Children in urban and rural areas in various european countries. Annals of Clinical Research 14: S33- 36.

29. Safrit, M (1990). The validity and reliability of fitness tests for children: a review. Pediatric Exercise Science 2(1): 9-28.

30. Sallis J, Hovell M, Hofstetter C, Elder J, Hackley M, Caspersen C, Powell K (1990). Distance between homes and exercise facilities related to frequency of exercise among San Diego residents. Public Health Rep 105(2): 179185.

31. Sallis J, Zakarian J, Hovell M, Hofstetter C (1996). Ethnic, socioeconomic, and sex differences in physical activity among adolescents. J Clin Epidemiol 49(2): 125-134.

32. SAS Institute (1990). SAS/STAT User's Guide. Cary, NC: SAS Institute.

33. Seabra AF, Maia JA, Mendonça DM, Thomis MA, Caspersen CJ, Fulton JE (2008). Age and sex differences in physical activity of Portuguese adolescents. Med Sci Sports
Exerc 40: 65-70.

34. Seabra AF, Mendonça DM, Thomis MA, Malina RM, Maia JA (2007). Sports participation among Portuguese youth 10 to 18 years. Journal of physical Activity and Health 4: 370380.

35. Silva M, Sobral F, Malina R (2003). Determinância sociogeográfica da prática desportiva na adolescência. Coimbra: Centro de Estudos do Desporto Infanto-Juvenil, Faculdade de Ciências do Desporto e de Educação Física. Coimbra: Universidade de Coimbra.

36. Taks M, Renson R, Beunen G, Claessens A, Colla M, Lefevre J, Ostyn M, Schueremans C, Simons J, Van Gerven D, Vanreusel B (1991). Sociogeographic variation in the physical fitness of a cross-sectional sample of Flemish girls 13 to 18 years of age. Am J Human Biol 3 (5): 503-513.

37. Tamura Y (1975). Physical fitness of rural adolescents. In: Asahina K, Shigiya R (eds). Human adaptability, vol. 4. Physiological adaptability and nutritional status of the Japanese. Tokyo: University of Tokyo Press, 50-61.

38. Tanner JM, Whitehouse RH, Cameron N, Marshall WA, Healy MJ, Goldstein H (1983). Assessment of skeletal maturity and prediction of adult height (TW2 Method). Oxford: Academic Press Inc. Ltd.

39. Vasconcelos MA, Maia J (2001). Actividade física de crianças e jovens - haverá um declínio? Estudo transversal em indivíduos dos dois sexos dos 10 aos 19 anos de idade. Revista Portuguesa de Ciências do Desporto 1: 44-52.

40. Wilczewski A, Salad M, Krawczyk B, Saczuk J, Majle B (1996). Physical development and fitness of children from urban and rural areas as determined by the EUROFIT test battery. Biology Sport 13: 113-126. 